\title{
La polilla Copitarsia decolora: revisión del complejo de especies con base en la morfología genital masculina y de los huevos (Lepidoptera: Noctuidae)
}

\author{
Andrés O. Angulo ${ }^{1} \&$ Tania S. Olivares ${ }^{2}$ \\ 1. Depto. de Zoología. Facultad de Ciencias Naturales y Oceanográficas, Universidad de Concepción, Concepción, \\ Chile; aangulo@udec.cl \\ 2. Casilla 4040 correo 3. Concepción, Chile; tolivare@udec.cl
}

Recibido 15-VI-2009. Corregido 07-XI-2009. Aceptado 08-XII-2009.

\begin{abstract}
The moth Copitarsia decolora: a review of the species complex based on egg and male genital morphology (Lepidoptera: Noctuidae). Copitarsia decolora is a widely distributed polyphagous species with a large number of nominal changes due to phenotypic plasticity. We reviewed 14 males from Mexico, Colombia, Venezuela, Peru and Chile and the literature produced between 2003 and 2008. An analysis of the clasper complex of C. decolora male genitalia was performed by standard dissection. Scanning electron microscope (SEM) photographs of eggs structures were used as taxonomic characters. The genus Copitarsia comprises 23 species. C. corruda is considered a synonym of $C$. decolora. Rev. Biol. Trop. 58 (2): 769-776. Epub 2010 June 02.
\end{abstract}

Key words: moths, eggs, male genitalia, subspecies, South America, new synonym, new combinations.

Copitarsia decolora (Guenée, 1852) es una especie polífaga de amplia distribución, con un importante número de cambios nominales debido a su plasticidad fenotípica (Angulo \& Olivares 2003). En 2005, la especie era conocida como Copitarsia turbata (Herrich-Schaeffer, 1855), al estudiar la sinonimia ordenada cronológicamente, se encontraron los siguientes problemas con el uso de C. turbata: 1. El espécimen tipo está perdido y 2. La localidad típica está errada ("Dalmatia"). Por lo tanto, es difícil verificar la identificación de la especie C. turbata debido a que no hay órganos genitales preservados ni localidad tipo claramente asociada al espécimen, además no corresponde al nombre más antiguo. Otro nombre propuesto es C. heydenreichii (Freyer, 1851) que corresponde a un homónimo de Agrotis heydenreichii (otra especie pero de la subfamilia Noctuinae); por lo tanto es recomendable evitar el uso de nombres iguales y cercanos. Finalmente, $C$. decolora es el nombre más antiguo y según el "principio de prioridad" es el que debe ser utilizado y se necesita una publicación que así lo acredite. Por estas razones Simmons \& Pogue (2004) proponen el uso del nombre C. decolo$\mathrm{ra}$, a pesar de los argumentos de Poole (1989) del "nomen conservandum" para mantener el uso de $C$. turbata ya conocida y utilizada por tanto tiempo. Por las razones expuestas se está realizando el cambio para lograr una estabilidad taxonómica, con una clara localidad tipo (para uso en agricultura) y con un espécimen tipo ( $C$. decolora tiene dos cotipos: uno en Londres y el otro en Paris).

Pogue \& Simmons (2008) caracterizan dos grupos de Copitarsia decolora (Guenée) (sensu lato) y proponen que el grupo con: "The clasper [lo cual corresponde al digitus] has concave, truncate apex" pertenece a $C$. decolora (Guenée) (sensu stricto) y el grupo con: "The clasper [digitus] has a straight to slightly convex apex" pertenece a Copitarsia corruda Pogue \& Simmons (2008). C. decolora es 
fitófaga en Alstroemeria sp., entre otros hospederos vegetales. La especie propuesta por Simmons y Pogue supuestamente no se encontraría en Alstroemeria sp. y se distribuye en Perú, Ecuador y Colombia; Simmons \& Pogue (2008) indican que hay una larva de Asparagus en un embarque de México interceptada en San Diego (California) por el USDA-APHIS.

Aquí hay dos aspectos para considerar: uno se relaciona con la forma del digitus y el otro con la distribución atípica de ese individuo larval en México, estos aspectos son relevantes para los estudios sistemáticos y taxonómicos de las especies del género Copitarsia.

Los caracteres típicos de los genitales masculinos de dicha especie permiten diferenciarla de su simpátrica $C$. incommoda, la cual se encuentra con distribución muy restringida en las alturas. El carácter que permite esta separación corresponde a la forma del digitus el cual para esta última es alargado (digitiforme).

Al preparar y analizar morfológicamente los genitales masculinos, se encuentra una gran variabilidad en el digitus, lo que permite sugerir a través del análisis morfológico algunos cambios taxonómicos y reordenar la nomenclatura correspondiente a las especies denominadas $C$. decolora (sensu lato). En el presente trabajo también se lleva a cabo el análisis morfológico de caracteres correspondientes al corion de los huevos. Este estudio permite establecer algunas consideraciones respecto a la identidad de $C$. decolora

\section{MATERIALES Y MÉTODOS}

Se revisaron 14 machos provenientes de México, Colombia, Venezuela, Perú y Chile; las fechas y el lugar de colecta aparecen en el material revisado, el cual se encuentra depositado en las Colecciones Científicas de la Universidad de Concepción (UCCC) (Apéndice I). Para la nomenclatura de las estructuras de los genitales masculinos se utilizó Forbes (1954) y Angulo et al. (1999) y se revisó la bibliografía producida entre los años 2003 y 2008. La microscopía electrónica de los huevos anali- zados se realizó según la metodología descrita por Angulo \& Olivares (1991).

\section{RESULTADOS}

Forma del digitus: en ejemplares de $C$. decolora de México se detectan tres variaciones del digitus en los genitales del macho (Fig. 1-3), además se han adicionado dos formas más para el material de Texcoco, Montecillo (Fig. 4-5), y se compara con la variación figurada en Suárez-Vargas et al. (2006) (Fig. 32) de material de Texcoco y del Rancho San Cristóbal de los Nava, Municipalidad de Acatzingo, Puebla.

Por otro lado en el material de Colombia (N. Quimbayo et al. en prep.) se distinguen dos variaciones en el digitus (Fig. 6-7), y también otras dos formas en el material proveniente de Jardines, Los Andes, Colombia (Fig. 8-9).

Las variaciones de México (Fig. 1) y la variación de Colombia (Fig. 6) corresponden a la descripción de C. corruda Pogue \& Simmons 2008.

Las variaciones en la forma de los digitus encontrados en Chile, van desde la forma crenulada para Santiago (Chile), anatiforme para Concepción (Chile) (Fig. 10-11 y 25-29) y lisa para Termas de Río Blanco (Chile) (Fig. 12).

Las formas que se encuentran en Ica (Perú) son similares a las encontradas en Concepción (Chile) (Fig. 13-15) y también a la forma crenulada que es compartida con Santiago (Chile) (Fig. 16).

La variación del digitus de Venezuela (Fig. 30) se asemeja a la de Perú (Fig. 31) pero difiere en el largo de la ampolla.

La variación de Colombia (Fig. 6) "The clasper [=digitus] has a straight to slightly convex apex" considerada por Simmons \& Pogue (2008) como la especie C. corruda se encuentra en Alstroemeria sp.

\section{Planta huésped: Alstroemeria $\mathbf{s p .}$}

Finalmente Pogue (2007), al revisar el género Psectrotarsia Dognin, 1907 (Heliothinae), transfiere dos especies de este género al 

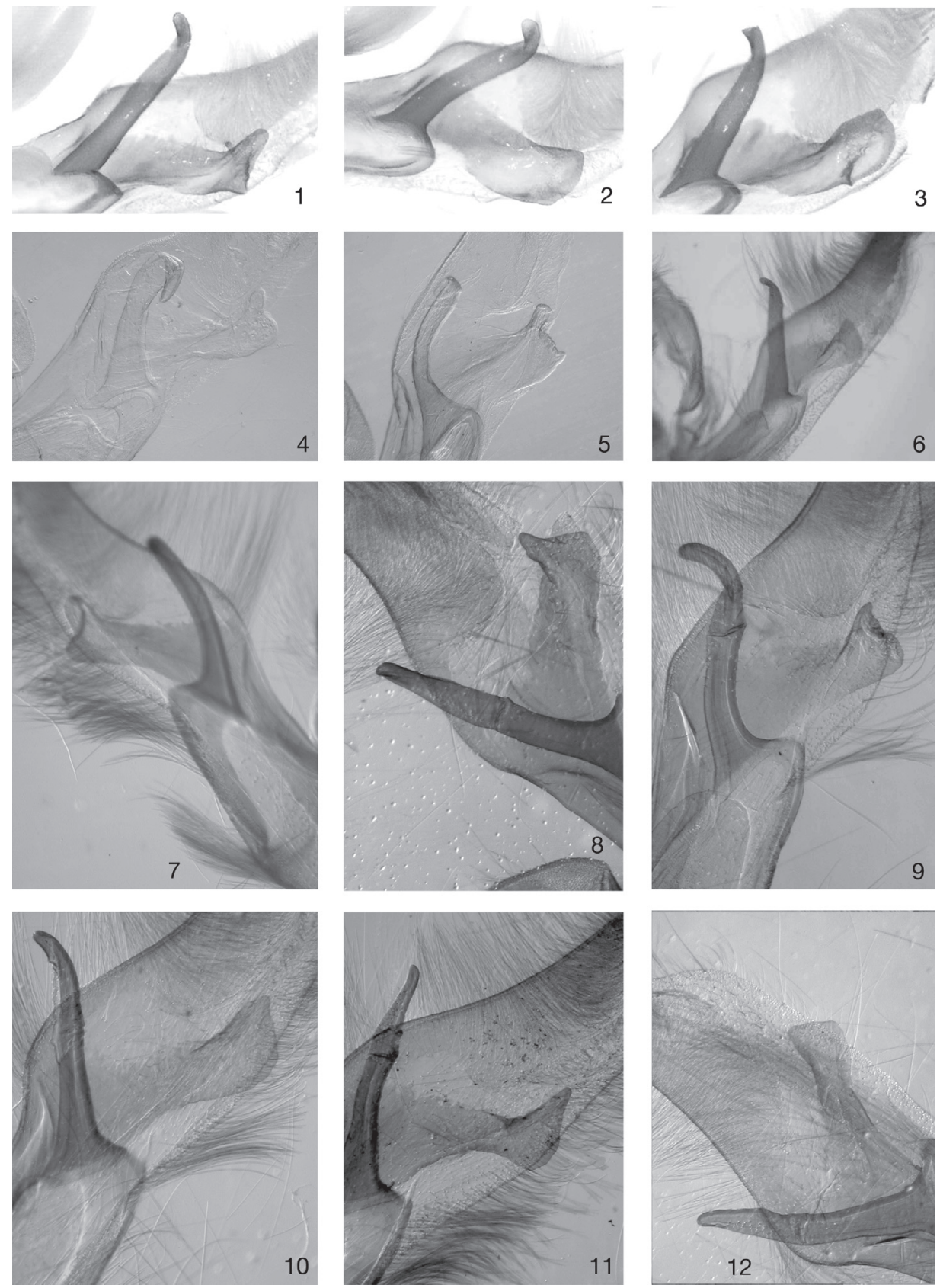

Complejo de la "prensilla" (clasper) de los órganos genitales del macho: 1-3. México (prep. D. Suárez); 4-5. México (Texcoco, Montecillo); 6-7. Colombia (prep. N. Quimbayo); 8-9. Colombia (Jardines Los Andes); 10-11. Chile (Concepción); 12. Chile (Termas de Río Blanco).

Clasper complex of male genitalia. 1A digitus variation in Copitarsia decolora from Mexico (Suárez-Vargas et al. (2006)). 

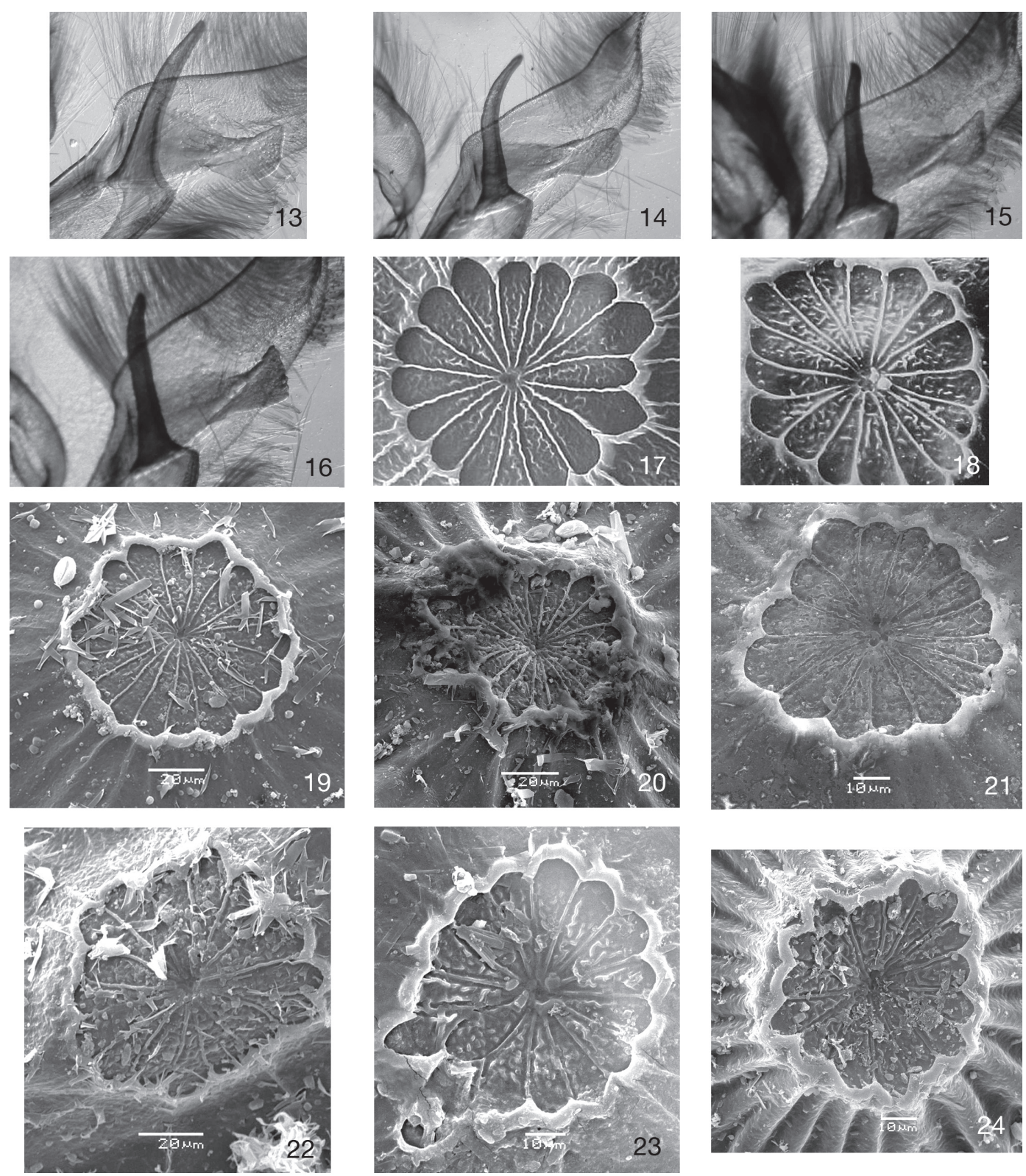

Complejo de la "prensilla" (clasper) de los órganos genitales del macho: 13-15. Chile (Concepción); 16. Chile (Santiago). Roseta primaria de huevos: 17. Chile (Chillán); 18. México (Texcoco); 19-24. Colombia (Jardines los Andes).

Clasper complex of male genitalia. 1A digitus variation in Copitarsia decolora from Mexico (Suárez-Vargas et al. (2006)). Primary egg rosette: 17 . 

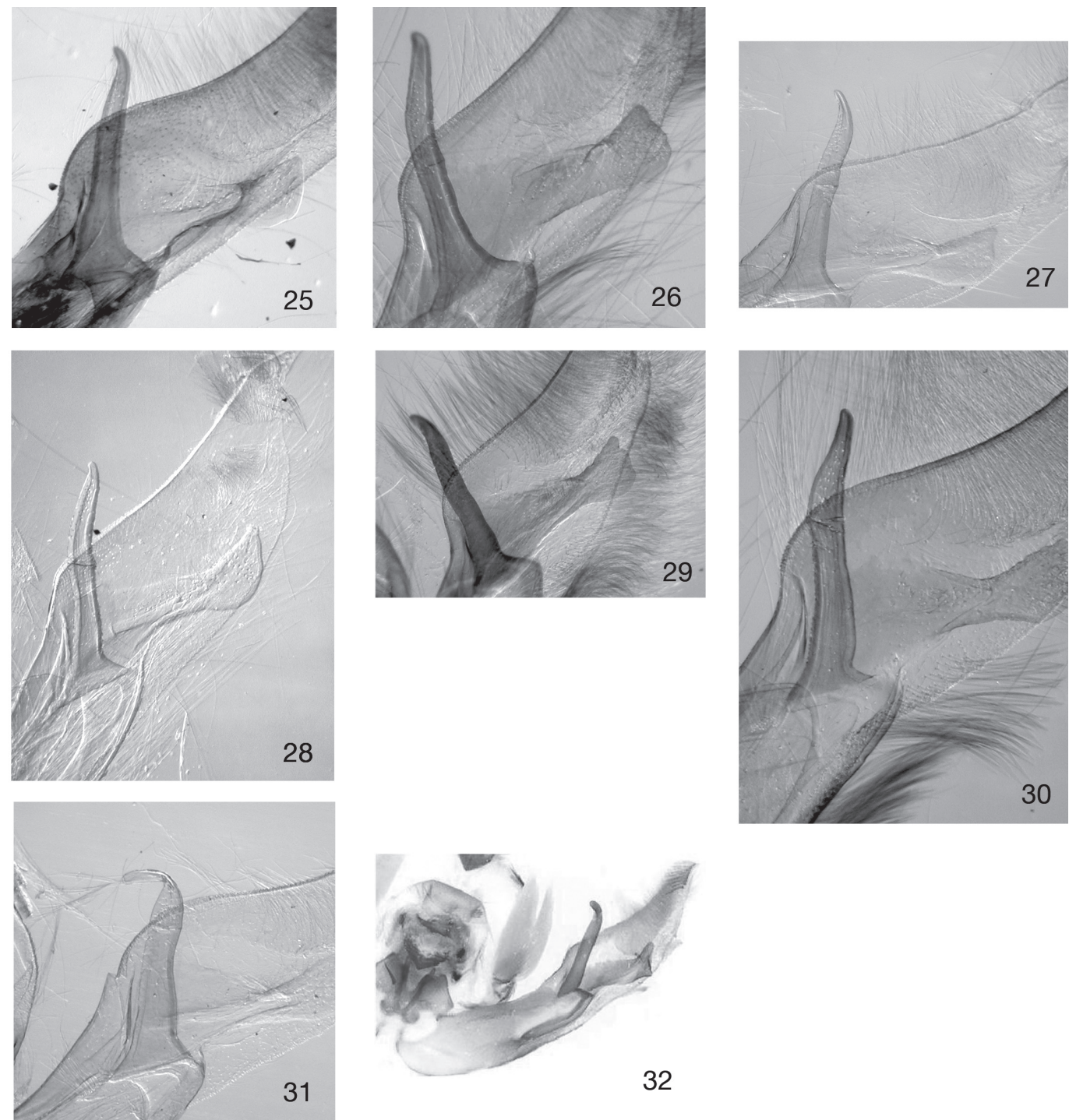

Complejo de la "prensilla" (clasper) de los órganos genitales del macho: 25. Chile (Concepción); 26. Chile (Azapa); 27. Chile (Chillán); 28. Chile (Coihueco); 29. Chile (Santiago); 30. Venezuela (Maracay); 31. Perú (Ica). 32. variación 1A del digitus de Copitarsia decolora en México (Suárez-Vargas et al., (2006)).

Clasper complex of male genitalia. 1A digitus variation in Copitarsia decolora from Mexico (Suárez-Vargas et al. (2006)). 
género Copitarsia Hampson, 1906: C. fuscirena (Hampson) y C. tamsi (Giacomelli); de tal manera que el catálogo de las especies de Copitarsia Hampson de Angulo \& Olivares (2003) queda actualizado de la siguiente manera:

\section{CATALOGO DE LAS ESPECIES DE COPITARSIA HAMPSON, 1906.}

COPITARSIA HAMPSON, 1906.

Copitarsia Hampson, 1906, p. 184.

Cotarsina Koehler, 1951, p. 166

Tarsicopia Koehler, 1952, p. 158. (in partim)

1. C. anatunca Angulo \& Olivares 1999.

2. C. anguloi Castillo 1991.

3. C. basilinea Koehler 1958.

4. C. belenensis (Koehler, 1973)

5. C. borsaniana (Koehler, 1958) n. comb

6. C. clavata Koehler 1955.

7. C. decolora (Guenée, 1852)

8. C. fleissiana (Koehler, 1958)

9. C. fuscirena (Hampson, 1910)

10. C. gentiliana (Koehler, 1961)

11. C. gracilis (Koehler, 1951)

12. C. humilis (Blanchard, 1854).

13. C. maxima (Koehler, 1961)

14. C. mimica Angulo \& Olivares 1999.

15. C. murina Angulo, Olivares \& Badilla, 2001.

16. C. naenoides (Butler 1882).

17. C. paraturbata Castillo \& Angulo 1991.

18. C. patagonica Hampson 1906.

19. C. purilinea (Mabille, 1885).

20. C. roseofulva (Koehler, 1952)

21. C. sulfurea (Koehler, 1973)

22. C. tamsi (Giacomelli, 1922)

23. C. vivax Koehler, 1951

\section{DISCUSIÓN}

Simmons \& Scheffer (2004) analizan secuencias del gen de la Citocromo oxidasa mitocondrial I (COI) para examinar la variación mitocondrial en $C$. decolora, evaluar y distinguir las diferentes razas de huésped o líneas crípticas: "we found that $C$. decolora is comprised of two distinct genetic lineages that are morphologically similar".
Debido a las variaciones encontradas en este estudio los linajes debieron considerarse subespecies, el estado taxonómico de Copitarsia decolora (Guenée) debería tener las siguientes opciones:

La primera opción corresponde a:

- Copitarsia decolora decolora (Guenée, 1852)

- Copitarsia decolora corruda (Pogue \& Simmons, 2008)

En este caso debe existir alo o parapatría en la distribución de las subspecies.

La segunda opción: Si hay distribución simpátrica de estas dos entidades, deberían ser sólo -a lo más- formas, pues serían el producto fenotípico de amplio rango de variabilidad genética de la especie. En esta segunda aproximación $C$. corruda se considera sinónimo junior de $C$. decolora.

La resolución final se obtendrá en un estudio de la distribución geográfica de este complejo dilucidando su alopatría o simpatría poblacional.

Con respecto a los caracteres del corion de los huevos, para Pogue \& Simmons (2008) $C$. corruda presenta una roseta primaria con 19 celdas. Para el caso de los huevos revisados en este trabajo, la variabilidad del número de celdas primarias está entre 10-13 (Olivares \& Angulo 2004), entre 16-17 (Artigas \& Angulo 1973) y los huevos revisados de Colombia entre 15 y 18 celdas. De lo anterior, se desprende que para diferenciar otras especies, se debe tomar en cuenta un mayor número de caracteres taxonómicos, como en este caso las celdas primarias del huevo.

\section{AGRADECIMIENTOS}

Al Proyecto $\mathrm{N}^{\mathrm{o}}$ 207.113.074-1.0 de la Dirección de Investigación de la Universidad de Concepción por el apoyo económico a la presente publicación, también hacemos extensivos los agradecimientos a Juan Cabrera La Rosa de la Universidad Privada Antenor Orrego, Trujillo, Perú por el envío de material biológico. 


\section{RESUMEN}

Copitarsia decolora es una especie polífaga ampliamente distribuida con un gran número de cambios nominales debido a su plasticidad fenotípica. Examinamos 14 machos de México, Colombia, Venezuela, Perú y Chile y revisamos la literatura producida entre 2003 y 2008. Realizamos un análisis del clasper (digitus) de los órganos genitales masculinos del complejo de C. decolora mediante una disección estándar. Utilizamos fotografías de microscopio electrónico de barrido (SEM) del corion de los huevos como carácter taxonómico. El género Copitarsia queda compuesto por 23 especies. C. corruda se considera un sinónimo de C. decolora (Guenée).

Palabras claves: huevos, nóctuido, genitales masculinos, subespecies, Sudamérica, nueva sinonimia.

\section{REFERENCIAS}

Angulo, A.O. \& G.Th. Weigert. 1977. Pseudaletia punctulata (Blanchard) y Pseudaletia impuncta (Guenée): nóctuidos hadeninos similares en Chile. Lepidoptera: Noctuidae). Agro Sur 5: 12-17.

Angulo, A.O. \& T.S. Olivares. 1991. Microestructura del exocorion en huevos de algunas especies de nóctuidos (Lepidoptera: Glossata: Noctuidae). Ann del Inst. Patagonia Serie Cs. Nat., Punta Arenas (Chile) 20: $95-100$.

Angulo, A.O., M.A. Rodríguez \& T.S. Olivares. 1999. Nuevo género y nuevas especies de nóctuidos altoandinos III. Faronta Smith, 1908 v/s Strigania Hampson, 1905 y algunos alcances acerca de Dargida Walker, 1856 (Lepidoptera: Noctuidae). Gayana 63: $67-85$.

Angulo, A.O. \& T.S. Olivares. 2003. Taxonomic update of the species of Copitarsia Hampson, 1906, (Lepidoptera: Noctuidae: Cuculliinae). Gayana 67: 33-38.
Artigas, J.N. \& A.O. Angulo. 1973. Copitarsia consueta (Walker) biología e importancia económica del cultivo de raps (Lepidoptera: Noctuidae). Bol. Soc. Biol. Concepción 46: 199-216.

Forbes, W.T.M. 1954. Lepidoptera of New York and neighboring states. Noctuidae. Part. III. Memoirs 329: $433 \mathrm{p}$

Olivares, T.S. \& A.O. Angulo. 2004. Descripción de los huevos de Copitarsia incommoda (Walker) y Copitarsia turbata (Herrich-Schaeffer) (Lepidoptera: Noctuidae: Cuculliinae). Gayana 68: 112-116.

Pogue, M.G. 2007. Revision of the genus Psectrotarsia Dognin, 1907 (Lepidoptera: Noctuidae: Heliothinae). Zootaxa 1637: 1-19.

Pogue, M.C. \& R.B. Simmons. 2008. A new pest species of Copitarsia (Lepidoptera: Noctuidae) from the Neotropical Region Feeding on Asparagus and Cut Flowers. Ann. Ent. Soc. America 101: 743-762.

Poole, R.W. 1989. Lepidopterorum Catalogus (N.S.) Fascicle 118. Noctuidae. Part. 1: 1-500.

Simmons, R.B. \& M.C. Pogue. 2004. Redescription of two often-confused noctuid pests, Copitarsia decolora and Copitarsia incommoda (Lepidoptera: Noctuidae). Ann. Ent. Soc. America. 97: 1159-1164.

Simmons, R.B. \& S.J. Scheffer. 2004. Evidence of Cryptic Species within the pest Copitarsia decolora (Guenée) (Lepidoptera: Noctuidae). Ann. Ent. Soc. America 97: 675-680.

Suárez-Vargas, A.D., N. Bautista-Martínez, J. ValdézCarrasco, A. Angulo-Ormeño, R. Alatorre-Rosas, J. Vera-Graziano, A. Equihua-Martínez \& V. ManuelPinto. 2006. Fluctuación poblacional de Copitarsia decolora (Guenée) y su asociación con crucíferas comerciales. Agrociencia (México) 40: 501-509. 


\section{APÉNDICE I}

Material de Copitarsia decolora proveniente de México, Colombia, Venezuela, Perú y Chile.

Material examinado (14 machos): Chile: 1 macho, Concepción, 24-xi-1958, trampas Coll (copi1); 1 macho, Chile, VIII región, Unimarc, Concepción, 6-xi-1997, Angulo Coll (copi 2); 1 macho, Azapa, Cerro Moreno, 13-xi-68, en malezas, ob. lab., R. Mendoza Coll. (copi 3); 2 machos, Termas de Río Blanco, Cautín, 3/51 (copi 7, copi 16); 1 macho, Concepción, 15-ii-58, J.A.C. Coll, cuncunilla betarraga (v); 1 macho, Tobalaba, 20-nov-46 (copi 14); 1 macho, Chillán, 24/12/80 (vv) (copi 13), 1 macho, Coihueco, 20 -Enero 1973, Moreno Coll (GGG) (copi 17) (UCCC). Colombia: 1 macho, Colombia, Cundinamarca, Bogotá, El Rosal, El Rodeo, finca Tingua Cardena. 04³5 '56“ N-740. $04^{\prime} 51^{`}$ W, 2 599m. 14 oct 2004, M Sosa. Fitófago en Rosa sp var. Vogue. Cultivo en estado vegetativo (copi 4); 1 macho, Colombia; Cundinamarca, Mun. Chía. Vda Fagua, M. G. Consultores $04^{\circ} 52^{\prime} \mathrm{N}-74^{\circ} 04^{\prime} \mathrm{W}, 2562$ m.s.n.m., cría de huevos de postura individual encontrados en cultivo de Alstroemeria sp. (copi 6); 1 macho, Colombia, 25-07-2007, C.I. Jardines de los Andes, Diana Navarrete Coll (copi 15) (UCCC). Venezuela: 1 macho: Dto. Federal Cumbre de Boquerón frente a bajo seco, 1 600m, 7-iii-1969, Coll N. Hung (con pupa) (copi 8) (UCCC). México: 1 macho, Chapingo, Méx, cultivo de col, Nestor Bautista M. 15/06/1992 (copi 9); 1 macho, Montecillo, Texcoco, cultivo de col, J.L. Velazco, 12/06/1998 (copi 10); 1 macho, Texcoco, Estado de México, Colegio de Postgraduados. Entomología agrícola, 27-08-2002, C. incommoda [error identificación], cría en lab., Roberto Flores. (UCCC). Perú: Fundo Galmos ICA, (espárrago progenitores, correspondiente a F1) crianza de laboratorio, UNMSM (copi12); 6 machos, Ica, 26/06/2008, L. Olortegui Coll (UCCC).

Huevos: seis huevos, Colombia, C. Jardines Los Andes, 25-07-2007, D. Navarrete Coll.; 4 huevos, México, Texcoco, Estado de México, Colegio de Postgraduados, Entomología Agrícola, 27-viii-2002, Bautista N. cría en Lab. (ex: col); 4 huevos, Chile, Chillán, Estación Experimental (INIA), viii-2002. M. Gerding, (ex: espárragos). 